\title{
Types of explanations students use to explain answers to conceptual physics questions
}

\author{
Anne T. Alesandrini and Paula R. L. Heron \\ Department of Physics, University of Washington, 3910 15th Ave NE, Seattle, WA, 98195
}

\begin{abstract}
In order to investigate student ability to use — and communicate — correct and complete reasoning, we examine written explanations from students in introductory university physics courses who have been prompted to answer a conceptual physics question and to "explain." Rather than focusing on context-specific reasoning difficulties, we examine the variation in what, to students, may constitute satisfying explanations, paying attention to what is present beyond what might score points on an instructor's rubric. We present six different types of explanations with examples that illustrate the breadth of student responses. This broad view of student explanations has the potential to guide instruction aimed at the development of student explanation and argumentation skills in ways that leverage and are responsive to how students initially explain their reasoning.
\end{abstract}




\section{INTRODUCTION}

When answering conceptual questions in physics, students are often asked for explanations to go with their answers. They are asked to "explain their reasoning," or more simply, to "explain." We as instructors and/or researchers may have a clear idea of what we are asking of them, but it is not clear that they understand the task in the same way that we do. For example, in the context of an exam or homework assignment, we may intend for introductory physics students to justify why the answers they have given are correct using physics principles, and we may score their explanations according to how well they accomplish this task. Some students offer responses that are well matched to this expectation, even if they are only partially successful in it, but others appear to be attempting something else entirely. In this research, we examine written explanations students give to support their answers to conceptual physics questions on homework and exams in the introductory physics classes at the University of Washington. We are interested not in the correctness of the content they offer, but in the variation that exists in the ways that students explain. In this paper, we will focus on different types of explaining that we find students do when asked to explain their reasoning.

We set out to investigate the ways that students explain their reasoning in order to gain insights that may prove helpful in improving instruction aimed at helping students explain their answers in ways that more closely match our expectations. We want our students to be able to use physics to create logical chains of reasoning that justify their answers. By knowing more about the explanations that our students give, we may be able to better tailor instruction to bridge the gap between where they are initially and where we would like them to be. This is somewhat analogous to the logic behind investigating students' prior conceptions of a particular physics topic; instruction that addresses the thinking our students are already doing can improve instructional outcomes [1,2]. Furthermore, if we consider student explanations that are submitted for grading to be a reasonable approximation of what they consider to be 'good reasoning,' or at least the most worthy of sharing of the reasoning they are doing on a given problem, our findings may provide insight into what students find satisfying in explanations or what they consider to be sufficient reasoning to reach, or accept, an answer.

Previous research has been done on the scientific explanations students construct to make sense of physical phenomena. For example, Yeo and Gilbert $[3,4]$ present a multidimensional framework that categorizes scientific explanations in terms of function, form, and level, which they apply in interview settings where high school students are asked to explain a specific physical phenomenon. Touger et al. [5] offer three categories for written student explanations to open-ended "what if..." questions: formuladriven, intuitive, and hierarchical. Formula-driven explanations proceed with algebraic manipulation from formulas; intuitive explanations use real-world terminology and are typically qualitative; hierarchical explanations use governing concepts and often involve multiple representations. The questions students are asked in such research are more broad and open-ended in scope than in our work, with the variable(s) to be analyzed largely unspecified. Instead of asking students to explain a phenomenon, our students are asked to answer a specific question and then explain that answer (see Fig. 1 for example questions).

Other research has been done on how students use argument in the science classroom using Toulmin's model of argumentation [6], where arguments are labeled and analyzed in terms of claims, warrants, grounds, quantifiers, rebuttals and backing. Arguments can then be fruitfully compared by attending to which of these features are present and what plays the role of each included or implied feature. While some of the explanations that students give in our research could very effectively be compared under such an argumentation framework, others are interesting precisely because they appear to be doing something other than making an argument. At this point we are more interested in the nature of students' own explanations than in the degree to which they diverge from those of "experts," or from some expected form.

\section{CONTEXT AND METHODS}

In this work, we examined the written explanations students gave in response to conceptual physics questions on homework and exams, post relevant instruction, in introductory physics classes at the University of Washington (UW). Questions were chosen in which no calculations were necessary and a minimal number of reasoning steps were required to reach the answers. The first set of explanations analyzed were from the first tutorial homework of the first quarter of the calculus- based introductory physics sequence on the topic of acceleration in one dimension [7]. Tutorial homework assignments are associated with the weekly tutorial sessions at UW, which supplement lecture instruction and allow smaller sections of students to develop conceptual understanding of physics topics in small groups. A sample of 93 student submissions of this first tutorial homework was collected from 5 tutorial sections chosen at random out of the total 26 sections taught that quarter. Examples of student explanations used in this paper are taken from this homework, and the relevant questions from this homework are included as Fig. 1. Students had received very minimal instruction and no formal feedback on what is expected for written explanations before completing this homework. We chose this as a starting place because we expected to see variation in explanations more plainly at this stage than in successive assessments.

As our aim is to capture the variation that exists in student explaining, a phenomenographic approach was taken [8]. In 
the analysis, more attention was paid to ways in which student explanations varied from each other than to ways in which they varied from what an "expert" might offer in the form of a solution. The initial emergent coding identified variation in the forms the explanations took (e.g., words, diagrams, equations), the features present in the explanations (e.g., specialized vocabulary, narrative elements, references to the physical situation of the problem or its representation), and the types of explaining present. Here we focus on the variation in types of explaining: the different ways in which an explanation supports an answer or connects that answer to other ideas. The next round of emergent coding, then, was used to group different types of explaining. These categories were revised after analyzing other homework and exam questions in different introductory physics courses at the University of Washington and in different physics contexts (primarily mechanics) in order to reflect categories that exist across different student populations and physics topics. A second coder was able to corroborate proposed categories on sample data before the categories reached this stage of finalization.

\section{from ACCELERATION IN ONE DIMENSION}

4. Two carts roll toward each other on a level table. The vectors represent the velocities of the carts just before and just after they collide.
Before

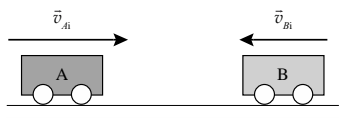

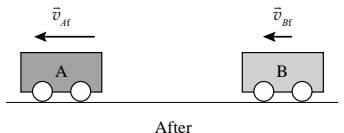

After a. Draw and label a vector for each cart to represent the change in velocity from before to after the collision. Make the magnitude and direction of your vectors consistent with the vectors drawn above. Show your work.

b. How does the direction of the average acceleration of cart A compare to the direction of the average acceleration of cart B over the time interval shown? Explain.

6. Carts A and B move along a horizontal track. The top-view strobe diagram below shows the locations of the carts at instants $1-5$, separated by equal time intervals.

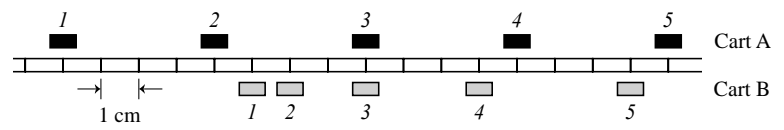

a. At instant 3 is cart A speeding up, slowing down, or moving with constant speed? Explain.

b. Is the speed of cart B greater than, less than, or equal to the speed of cart A at instant 3? Explain.

FIG. 1. Questions from the homework. Note: When responding to question $4 \mathrm{~b}$, students will have already determined change-invelocity vectors for both carts.

In line with a phenomenographic approach, the intention is not to categorize students by means of their explanations, but rather to categorize the ways of explaining that are evidenced in the pool of student explanations as a whole. Thus, individual students may do more than one type of explaining within a single homework or within a single explanation. We are also not, at this point, interested in the relative frequency of these types, but rather a depiction of the variation that is present.

\section{PRELIMINARY RESULTS}

What follows is a list of types of explaining that we find students are doing in their written explanations. These types reoccur, demonstrate the breadth that exists within type, and may inform future research and instruction. More than one type of explaining is often present in a single explanation. All explanation types may be used in support of either a correct or incorrect answer. (Please refer back to Fig. 1 for the questions used in the examples.)

(1) "You might think" explanations - These center around features of the question that might tempt someone else to come to a different conclusion than the responder has. Rather than staying limited to why a chosen answer is correct, these may explore why a different answer is incorrect. Example 1, in response to homework question 6b: "Less than because even though they are in the same position at the same time, cart B started at a different position than cart A and cart A is still increasing its displacement faster than cart B." Here, the student is pointing out that the carts "are in the same position at the same time," not because their own answer depends on it, but to acknowledge its irrelevance to their answer. They answer as they do "even though" this feature is present, that is, in spite of whatever competing answer this feature might suggest.

(2) Process explanations - These describe the steps taken, often in abstract form, to arrive at an answer. They do less to justify the answer directly than to offer the reader a process by which the answer can be found. Example 2, in response to homework question $6 \mathrm{~b}$ : "Less than. If you draw a line from instant two to instant three for both cart A and B, then the line would be longer for A." Here, the student is instructing us what we need to do ("draw a line from instant two to instant three for both cart A and B") in order to determine the answer as they have.

(3) Contextualizing explanations - These take an approach beyond the physics topic at hand. They might describe what causes the answer to be true, relate the outcome to a potentially analogous 'real world' situation, or use previously learned physics concepts. In doing so, they bring in a broader context that justifies or corroborates the answer. (Note: The following example responses came before any instruction of forces had occurred in this course or its prerequisites.) Example 3A, in response to homework question $4 b$ : "They go in opposite directions, as the collision pushed them apart." This student tells us what causes their answer to be true ("the collision pushed them apart"), rather than explaining in terms of kinematics. The degree to which this student's notions of pushes impacting motion is based on formal dynamics instruction from a previous physics course or from intuition from real-life experience is unclear. Example 3B, in response to homework question 4b: "The acceleration directions are opposite because a force coming 
from the opposite direction caused them to slow down/ go the other way." Here, the student sees the accelerations of the carts as caused by forces, which they likely learned about in a previous physics course. Their answer, then, is related to a larger context that goes beyond the instruction they have so far received in class.

(4) "Expert" explanations - These include statements of what physics principles were used to arrive at an answer, how those physics principles are relevant to the situation presented in the question, and therefore what the answer must be. For the purposes of this research, an explanation is considered this type if it is structured in this way, whether or not a correct answer has been reached. An explanation offered as a full-credit solution by the instructor would likely be of this type. Example 4A, in response to homework question 4b: "Since average acceleration is the change in velocity divided by the time interval, carts A \& B have an opposite direction of change in velocity, therefore the direction [of] their average acceleration is opposite as well." This student states a relevant definition for the quantity they are asked about ("average acceleration is the change in velocity divided by the time interval") and connects it to the problem at hand ("carts A \& B have an opposite direction of change in velocity") to justify their answer. Example $4 B$, in response to homework question 6a: "Cart A moves with constant speed. Cart A is consistently $4 \mathrm{~cm}$ apart for each time interval. $\Delta$ position $/ \Delta$ time is the same, so velocity, and subsequently speed, is constant." This student identifies a feature of the scenario ("Cart A is consistently $4 \mathrm{~cm}$ apart for each time interval"), then relates it to the quantity being asked about (" $\Delta$ position/ $\Delta$ time" and so velocity and speed) in order to justify their answer.

(5) Salient features explanations - These identify features of the scenario (e.g., types of motion, events, comparisons between variables) that are offered in support of the answer. While many explanations include features of the scenario as part of a larger explanation, and therefore could be said to contain this kind of explaining, some explanations are remarkable because they include nothing else. For example, these explanations may be like "expert" explanations that fail to mention the underlying reasons why these features connect to the answer given. Example 5A, in response to homework question 4b: "The [directions of the average accelerations are] opposite since cart A changed direction but cart B didn't change direction." Here, the student has identified whether each of the carts has changed directions or not, which is relatively easy to see from the question prompt, as the feature that leads to their answer. No more justification is given. Example 5B, in response to homework question $4 b$ : "The Ave acceleration of A cart should be [in the] same direction as cart B cuz they move in same direction after [the] collision." This student identifies the final direction of motion of the two carts, which is given in the diagram, as the feature that leads to their answer. No more justification is given. Example 5C, in response to homework question 6a: "Constant speed, the strobe carts are evenly spaced." This student points out the spacing of the images of the cart apparent in the question diagram. The relevance of this feature is left unexplained.

(6) Description explanations - These tell more about the answer, perhaps giving other details about a variable in question or about what happens next, focused on taking us beyond the answer more than how the answer was determined or why it makes sense. Example 6, in response to homework question 4b: "A's average acceleration is negative, B's is also negative but smaller." Rather than simply state their conclusion that the average accelerations are both in the same direction, this student supplies additional information about those accelerations, namely, that both are negative and that one is smaller than the other. This provides more details about the variable in question without describing how any of this is known.

\section{DISCUSSION AND FUTURE RESEARCH}

There is considerable breadth in the types of explaining students include in their written explanations. The mode of explanation we as instructors typically aim for, that of connecting physics principles to the particulars of a question prompt and demonstrating how they lead to a particular answer, is one type out of many.

We assume that all students did more thinking than what they wrote down in their explanations, and our analysis is purposefully limited to what was written explicitly. That is, we envision the thinking that a student does when determining an answer and the explaining that a student does when writing an explanation as two distinct, although interrelated, processes. As such, there are likely interesting complexities in student reasoning hiding within individual explanation types. In particular, those students who gave salient features explanations are likely to have used different types of reasoning to connect the features they mention to the answers they give. What they have in common is their choice not to put that part of their reasoning down in writing. A next stage of research will be conducting student interviews to investigate how students are reasoning as they make an answer choice compared to what they chose to write down. We also hope to gain insight into the degree to which they are actively considering their instructor audience as they write an explanation as well as what students value or find lacking in particular explanations.

As instructors, we see value in many of the student explanation types, even as we hope that students who provide explanations of these types can additionally give us "expert" explanations. For example, "You might think" explanations are powerful in refuting what might be a tempting intuitive choice; being able to talk oneself out of those answers, or explain to a classmate why the seemly obvious choice is not correct, is also something we would like our students to be able to do. Process explanations reflect important knowledge about the mechanics of completing a problem that we often offer to our students at 
some point during instruction and that students might find useful in offering to each other. Contextualizing explanations are ones we might hope that our students use to predict an answer or to double check an answer that has been justified with an "expert" explanation. In this way, contextualizing explanations can help our students incorporate knowledge from one physics topic into a larger framework that spans other parts of physics as well as life outside of the classroom. Our purpose need not be, then, to eliminate these types of explaining from our students' repertoires. Instead, similar to the approach of a resources framework for conceptual understanding [9], we may consider the types of explaining that our students are already doing as something that we as instructors can build upon. Our instructional goals might then be to contextualize when and where these different forms of explanations might be advantageous and where they may fall short of what is required. Simultaneously, we might also aim to make clear how powerful we find "expert" explanations to be and make the case for why we consider an "expert" explanation the best choice in the contexts that we require it (or grade based on it) in our courses. Further investigation of the utility of this approach is possible.

An additional argument for addressing these different types of explaining more explicitly in classroom instruction comes from the work being done using dual process theories of reasoning [10-13]. According to this framework, a heuristic process is always our starting place in approaching a problem, and necessarily, our intuitions influence the outcomes of our thought processes. A second, analytic process only comes into play to the degree that it seems necessary to justify, check, or override our initial intuitive response. Helping students to know, then, when the reasoning they have done is sufficient to give them wellearned confidence in their answers is a worthy goal. For example, a salient features explanation might be enough to justify an answer with high confidence in a topic area where a person has developed expert intuition (that is, where their heuristic process is likely to be correct). However, a student might be clued in to a need to do additional thinking in order to be sure of an answer if they find they are only able to supply a salient features explanation in a topic area where they do not have such expertise (that is, where their heuristic process is just as likely to be incorrect). Additionally, acknowledging the role that other non-"expert" explanations might play in all of our thought processes and making space for them in our classroom conversations might positively impact student metacognition in terms of awareness of the strengths and pitfalls present in their own thought processes.

Further investigation could also center around the generalizability and prevalence of different types of explaining over time, across different student populations, physics topics, and/or question types. We will also be investigating how instructors and graders explain their own reasoning, including the extent to which the explanations produced by these "experts" fall into the category which we have preliminarily called "expert" explanations. Additionally, we will investigate their views of student explanations.

\section{ACKNOWLEDGMENTS}

We wish to thank the participants of the 2019 FFPER Graduate Student / Postdoc Symposium for their helpful feedback. This work was supported in part by NSF grant DUE-1821123.
[1] P.S. Shaffer and L.C. McDermott, Research as a guide for curriculum development: An example from introductory electricity. Part II: Design of instructional strategies, Am. J. Phys. 60, 1003 (1992).

[2] N. D. Finkelstein and S. J. Pollock, Replicating and understanding successful innovations: Implementing tutorials in introductory physics, Phys. Rev. ST Phys. Educ.Res. 1, $010101(2005)$.

[3] J. Yeo and J. K. Gilbert, Constructing a Scientific Explanation - A Narrative Account, Int. J. Sci. Educ. 36, 1902 (2014).

[4] J. Yeo and J. K. Gilbert, in Multiple Representations in Physics Education edited by D. F. Treagust, R. Duit, and H. E. Fischer (Springer, Cham, 2017), pp. 255-287.

[5] J. S. Touger, R. J. Dufresne, W. J. Gerace, P. T. Hardiman, and J. P. Mestre, How novice physics students deal with explanations, Int. J. Sci. Educ. 17, 255 (1995).

[6] S. E. Toulmin, The Uses of Argument (University Press, Cambridge, 1958).

[7] L. C. McDermott and P.S. Shaffer, Tutorials in Introductory Physics, Updated Preliminary Second Edition (Prentice Hall, Upper Saddle River, NJ, 2011).
[8] F. Marton, Phenomenography - Describing conceptions of the world around us, Instr. Sci. 10, 177 (1981).

[9] J. P. Smith III, A. A. diSessa, and J. Roschelle, Misconceptions Reconceived: A Constructivist Analysis of Knowledge in Transition, J. Learn. Sci. 3, 115 (1994).

[10] D. Kahneman, Thinking, Fast and Slow (Farrar, Straus and Giroux, New York, 2011).

[11] M. Kryjevskaia, M. R. Stetzer, and N. Grosz, Answer first: Applying the heuristic-analytic theory of reasoning to examine student intuitive thinking in the context of physics, Phys. Rev. ST Phys. Educ. Res. 10, 020109 (2014).

[12] A. F. Heckler, The Ubiquitous Patterns of Incorrect Answers to Science Questions: The Role of Automatic, Bottom-up Processes, Psychol. Learn. Motivation, Adv. Res. Theory 55 , 227 (2011).

[13] C.R. Gette, M. Kryjevskaia, M.R. Stetzer, and P.R.L. Heron, Probing student reasoning approaches through the lens of dual-process theories: A case study in buoyancy, Phys. Rev. Phys. Educ. Res. 14, 010113 (2018). 